\title{
Hábitat y desarrollo. Explorando caminos de innovación educativa en arquitectura y urbanismo
}

\author{
Habitat and development. Exploring paths of educational \\ innovation in architecture and urbanism
}

\author{
Manuel J. Martín Hernández* y Vicente J. Díaz García** \\ Fecha de recepción: 03-04-2017 - Fecha de aceptación: 23-06-2017 \\ Hábitat y Sociedad (ISSN 2173-125X), n. ${ }^{\circ}$ 10, noviembre de 2017, pp. 113-128. \\ http://dx.doi.org/10.12795/HabitatySociedad.2017.i10.07
}

\begin{abstract}
Habitat and development. Exploring paths of educational innovation in architecture and urbanism. It has been two decades since the first subjects that incorporated basic habitability or development cooperation appeared in the training of the architect in Spain. Since then the schools of architecture have been incorporating content that is increasingly demanded by both society and students themselves. The economic crisis initiated in 2008 or the Sustainable Development Objectives approved in 2015 by the United Nations should make us reflect on the centrality of these issues in the formation of architecture and urbanism. Since the year of its creation, in 2001, the optional Habitat and Development has been proposed to make visible the social architecture as an important part in the architect's training, in which the architectural process is prioritized, closely related to the habitat (the inhabitant, the habits and their relation to the place), in front of the product (the object, the building, as a formal synthesis of that architecture). The subject makes a reading of the role that development cooperation can play in the training of the architect. Citizen participation is another of the cross-cutting themes that are incorporated into the subject by training students in techniques specifically applicable to the field of architecture and urbanism. Habitat and development is taught in the first semester of fifth year of the degree in architecture of the University of Las Palmas de Gran Canaria.
\end{abstract}

Key words

Habitat, Development cooperation, Social architecture, Citizen participation, Urban mediation

\section{Resumen}

Han pasado dos décadas desde que aparecieron las primeras asignaturas que incorporaban la habitabilidad básica o la cooperación al desarrollo en la formación del arquitecto en España. Desde entonces, las escuelas de arquitectura han ido incorporando contenidos que son cada vez más demandados, tanto por la sociedad como por los propios alumnos. La crisis económica iniciada en 2008, o los Objetivos de Desarrollo Sostenible aprobados en 2015 por Naciones Unidas, nos deben hacer reflexionar sobre la centralidad de estos temas en la formación de la arquitectura y el urbanismo. Desde el año de su creación, en 2001, la asignatura optativa Hábitat y desarrollo se ha propuesto visibilizar la arquitectura social como una parte importante en la formación del arquitecto, en la que se prioriza el proceso arquitectónico, íntimamente relacionado con el habitar (el habitante, los hábitos y su relación con el lugar), frente al producto (el objeto, el edificio, como una síntesis formal de esa arquitectura). En la asignatura se hace una lectura del papel que la cooperación al desarrollo puede jugar en la formación del arquitecto. La participación ciudadana es otro de los temas transversales que se incorporan en la asignatura, formando a los estudiantes en técnicas específicamente aplicables al campo de la arquitectura y del urbanismo. Hábitat y desarrollo se imparte en el primer semestre de quinto curso del grado en arquitectura de la Universidad de Las Palmas de Gran Canaria.

\section{Pallabras clave}

Hábitat; Cooperación al desarrollo; Arquitectura social; Participación ciudadana; Mediación urbana

\footnotetext{
* Catedrático jubilado de la Universidad de Las Palmas de Gran Canaria y profesor huésped del CUAAD de la Universidad de Guadalajara, México.

** Doctor arquitecto, profesor ayudante doctor del grado en arquitectura de la ULPGC. Universidad de Las Palmas de Gran Canaria, Escuela de Arquitectura. Campus de Tafira, Las Palmas de Gran Canaria. vicente.diaz@ulpgc.es.
} 


\section{Sobre la asignatura}

En un mundo cada vez más interconectado el arquitecto debe saber acercarse a otras culturas y distintas realidades sociales con curiosidad y ganas de aprender. En un momento en el que cada vez se demanda una mayor especialización de los profesionales, el arquitecto debe seguir aportando una visión de conjunto que sea capaz de conjugar e integrar, esto es, componer ${ }^{1}$ la complejidad que le rodea. En las asignaturas de teoría e historia, a lo largo de los diferentes cursos del grado en arquitectura, los estudiantes se acercan a los conceptos básicos de la disciplina, así como a sus procesos de producción. ${ }^{2}$ Ya en quinto curso, antes de su graduación, con la asignatura optativa Hábitat y desarrollo $(\mathrm{HyD})$, pueden también abrirse a un campo de experimentación profesional en esa arquitectura social que comprende conceptos como habitabilidad básica, participación ciudadana o cooperación al desarrollo.

Con respecto al estado de la cuestión, el pasado y el presente de la docencia en cooperación al desarrollo en las escuelas de arquitectura españolas está vinculado a personas concretas que han puesto su empeño en incluir estos contenidos en las diferentes materias de las que son responsables. Empezando por Julián Salas y Felipe Colavidas, desde la Universidad Politécnica de Madrid, con la creación a finales de la década de los noventa de una asignatura de doctorado (1996), así como el "curso de especialización en cooperación al desarrollo de los asentamientos humanos" (1997). En Barcelona, Pedro Lorenzo, con la asignatura "Arquitectura y Cooperación Internacional" en la ETSA del Vallés, y Raimón Torres en 1998, con las asignaturas optativas "Vivienda y cooperación" y "Tecnologías de bajo costo para la cooperación" (asignaturas que actualmente coordina Sandra Bestraten) en la ETSA de Barcelona; en 2001 iniciábamos la andadura de "Hábitat y desarrollo" en la escuela de arquitectura de Las Palmas de Gran Canaria, y en Sevilla, Esteban de Manuel, ponía en marcha la asignatura homónima, así como el Máster en Gestión Social del Hábitat; en la Universidad de La Coruña, Plácido Lizancos, con la asignatura "Análisis Arquitectónico", y Jorge Rodríguez, con la asignatura "Hábitat Básico"; en Alcalá de Henares, Paz Núñez y Roberto Goycoolea, tanto en el grado de arquitectura como a través de asignaturas de Máster como "Intervención en la ciudad no planificada" y "Construcción social del hábitat". Aunque son muchos los que quedan fuera, también podemos citar a Josep María Llop desde la Universidad de Lleida o a Carmen Mendoza desde el Máster de Cooperación Internacional de la Universidad Internacional de Cataluña, a Isabel Raposo desde la Facultad de Arquitectura de la Universidad de Lisboa o Enkarni Gómez, Alex Mitxelena o Izaskun Aseguinolaza en la Escuela de Arquitectura de San Sebastián. ${ }^{3}$

Frente a una arquitectura centrada en edificios o conjuntos paradigmáticos, o en sus firmantes, HyD trata de acercar al alumno a un conocimiento y una arquitectura, no menos importante, que se desarrolla en los márgenes, bordes, límites o fronteras culturales, disciplinares o del pensamiento. En la sociedad española, y también en la arquitectura, estamos actualmente experimentando uno de los extremos de esa rueda de la fortuna de la que hablaban los intelectuales colombianos en el año 2001. ${ }^{4}$ En el programa de HyD, centrado en lo que denominamos arquitectura social, se reflexiona sobre una arquitectura que trata de dar respuestas a la pobreza, a las desigualdades, a las emergencias. ${ }^{5}$ Se trata de problemas a los que casi siempre llegamos tarde, vinculados 
con ese otro $90 \%$ de la población a la que apenas prestamos atención: en el interior de los barrios marginales, en las periferias, en las catástrofes naturales y humanas. Es por ello que creemos en la necesidad de impartir una formación que nos permita actuar allí donde esta urgencia de la arquitectura así lo requiera.

Uno de los objetivos de la asignatura busca demostrar, tanto en la teoría como en la práctica, que la formación de arquitecto - y estamos en una asignatura del último curso del grado en arquitectura- debe permitir encarar la práctica profesional con garantías de inserción social y laboral a partir de dos cuestiones: una que tiene que ver con la diferencia,${ }^{6}$ esto es, con aquello que caracteriza la formación de la arquitectura frente a otras disciplinas; y la segunda, más práctica, consiste en mostrar ejemplos de arquitectos y arquitecturas que están trascendiendo las fronteras de lo más disciplinar o "científico-técnico" de la disciplina.

En los largos años sesenta se comenzó a dibujar un nuevo camino para la arquitectura. Paralelamente a la proliferación de una crítica creciente, especialmente desde otras disciplinas, ${ }^{7}$ la arquitectura también comenzó a ofrecer alternativas al modelo implantado y extendido tras la segunda guerra mundial por la arquitectura moderna, los CIAM y el denominado "Estilo Internacional". Ese nuevo camino incorporaba dos nuevos temas: por un lado, la ecología, esto es, el respeto por el lugar y los ecosistemas; y por otro, la participación ciudadana, o lo que es lo mismo, la importancia de los procesos sociales en la creación de la ciudad y del hábitat.

En HyD tratamos de hacer una relectura de aquellas propuestas, adaptándolas a las actuales circunstancias y, en especial, formando al alumnado en el uso de las herramientas metodológicas y técnicas que permitan su aplicación en la ciudad actual. También pretende traer una reflexión teórico-práctica sobre la vivienda y sobre la realidad social y cultural que nos rodea, tanto en nuestro entorno insular como respecto a nuestros vecinos de África o América latina. Los problemas a los que nos enfrentamos como arquitectos, con respecto al hábitat, a la ciudad o al territorio, no distan demasiado de aquellos a los que se deben enfrentar los arquitectos de otras geografías. Sin embargo, la afección de esos problemas, el tiempo de actuación o los medios de los que disponemos son diferentes según el momento en que los abordemos.

El programa de la asignatura pretende relacionar la realidad de la cooperación al desarrollo de asentamientos humanos en América latina y África con la realidad que existe en Canarias con respecto, por ejemplo, a la vivienda social o a la vivienda de autoconstrucción. De esta forma, se pretende incidir en una universalidad de los problemas que nos legitima, como arquitectos, para intervenir. Por último, se estudia la formación del arquitecto y su relación con el resto de disciplinas sociales (antropología, geografía, sociología...) en un marco general denominado "Arquitectura Social". La asignatura se estructura en dos bloques teóricos: por un lado, el hábitat, que se orienta hacia el estudio de la habitabilidad básica, del hábitat informal, de la vivienda social o del hecho de habitar. Por otro lado, el desarrollo, que se orienta hacia el estudio del aumento y la incidencia de las desigualdades, de la búsqueda del desarrollo humano, así como destacar el papel de la cooperación al desarrollo en la arquitectura. El programa de HyD se complementa con dos ejes transversales prácticos relacionados con la participación y con la gestión social del hábitat. El primer eje transversal se refiere a la introducción de la participación ciudadana en la forma- el premio Pritzker). Así, entre el 12 de febrero y el 1 de mayo de 2006 el MoMA celebró la exposición "Arquitecturas emergentes”; cinco años después, entre el 3 de octubre de 2010 y el 3 de enero de 2011, le siguió la exposición "Pequeña escala, gran cambio: la nueva arquitectura de lo social" (Small Scale, Big Change: New Architectures of Social) y en 2012, "Diseña con el otro 90\%". También en Viena tiene lugar la exposición "Piensa global, construye social". Podemos también destacar otras exposiciones posteriores como la del MoMA de 2014 "Uneven growth: tactical urbanism".

6 En parte podríamos relacionarla con la différance de Jacques Derrida y las teorías de la deconstrucción y específicamente con "la existencia de intervalos que evitan la relación unívoca entre la regla y los usos particulares de esa regla”. Para más información ver Martín Hernández, M. (1997, pp. 25-27).

7 Baste citar las aportaciones desde la sociología de Jane Jacobs (1961), Françoise Choay (1965), Henry Lefebvre (1968) o Manuel Castells (1973); desde la antropología de Lévi-Strauss (1962) o Marvin Harris (1962); desde la semiología de Roland Barthes (1961) o Umberto Eco (1962) y desde otras disciplinas de Guy Debord (1958), Ezequiel Ander Egg (1962), Raymond Queneau (1966), Edgar Morin (1969) o Murray Bookchin (1974), entre otras. 
ción del arquitecto a través del aprendizaje de técnicas participativas, así como de la aplicación práctica de las mismas. El segundo eje tiene que ver con la ciudad y el urbanismo emergente, con la educación de la mirada y con la forma en que nos acercamos a esa ciudad. En este caso se concreta en la realización de paseos, a modo de clases, por diferentes lugares de la ciudad.

Si abordamos el problema de abajo arriba, podemos trasladar esa idea, propuesta por el historiador Stefan Zweig en su libro Momentos estelares de la humanidad (1927), de la existencia, en el interior de la historia, de pequeños momentos que decantan a un lado $\mathrm{u}$ otro la balanza (Zweig, 2002). Al igual que en el campo de la economía o de la cooperación al desarrollo en la conferencia de Bretton Woods, en 1944, se trazaron las líneas de acción de la economía mundial después de la segunda guerra mundial, también en la arquitectura encontramos esos instantes estelares, como la publicación de la Carta de Atenas en 1943, redactada por Le Corbusier como una interpretación muy particular de lo acontecido en el IV CIAM dedicado a la Ciudad Funcional y desarrollado entre Marsella y Atenas, en 1933 (Montes Serrano, 2000), casi al mismo tiempo que se inventaba un supuesto "Estilo Internacional" a través de la exposición homónima celebrada en el MoMA de Nueva York en 1932. Aunque en 1961 la activista Jane Jacobs ya expresara una certera crítica sobre la destrucción de la ciudad tradicional, aquellas ideas de ciudad y de "estilo" que habían contribuido a fraguar un cambio de paradigma en lo económico, y también en lo arquitectónico, seguirían dominando una parte importante del pensamiento y la acción pública de las siguientes décadas.

De la misma manera podemos presuponer que en este momento, en algún lugar del planeta, está sucediendo algo que va a marcar un antes y un después para la arquitectura. De hecho, algunos autores se refieren a este momento como el de la gran aceleración, en referencia a un conjunto de estudios y teorías que plantean que nos encontramos ante un nuevo momento estelar de la humanidad: una era "antropocénica" (Steffen et al., 2015). Las propuestas del decrecimiento de Serge Latouche (2011) o Carlos Taibo (2017), las ciudades en transición, etc., incluso el cisne negro de Nicholas Taleb (2011), irían también en esa dirección. Juhani Pallasmaa (2015), citando a David Harvey, Fredric Jameson o Paul Virilio, nos habla de la desaparición del tiempo experiencial en la arquitectura del siglo xx: "Mientras que los edificios y los lugares construidos antes de la modernidad eran documentos de un tiempo benevolentemente lento, la arquitectura parece haberse vuelto más rápida, apresurada e impaciente a lo largo de la era moderna" (Pallasmaa, 2015, p. 116).

Junto a esos procesos de aceleración entrópica, también es acelerado el surgimiento de procesos en el sentido contrario. En diferentes escuelas de arquitectura de todo el mundo proliferan las iniciativas que dan importancia a la sostenibilidad, al reciclaje o a la participación ciudadana (Franco, 2016). Se trata de un fenómeno complejo y que denota una crisis de la propia disciplina. En su novela Mr. Gwyn, Alessandro Baricco (2011) nos presenta la idea de un escritor que renuncia a la tradicional pretensión de todo autor, al menos desde Guttenberg, de difundir su obra, para concentrarse en el oficio del retrato escrito. ${ }^{8}$ La idea de la artesanía también ha sido rescatada por Richard Sennett (2009) como una forma de resistencia a la tiranía de los sistemas expertos teorizados por Anthony Giddens (1999).

$\mathrm{Si}$ abordamos el problema de arriba abajo debemos hablar de la 
oportunidad que representa para la arquitectura la aprobación en 2015 por parte de las Naciones Unidas de los Objetivos de Desarrollo Sostenible (ODS, 2015-2030). A diferencia de lo que sucedía con los Objetivos de Desarrollo del Milenio (ODM, 2000-2015), que se proponían para su aplicación en los países con menor desarrollo humano, los ODS conciernen a todos los países del planeta. Concretamente, el objetivo 11 propone "lograr que las ciudades y los asentamientos humanos sean inclusivos, seguros, resilientes y sostenibles", persiguiendo, entre otras cosas, "participar activamente en la gobernanza y la gestión de tu ciudad" y "tomar nota de lo que funciona y de lo que no funciona en tu comunidad" (ONU, 2015).

\section{Sobre el hábitat}

En lo que se refiere al hábitat trazamos una historia de ida y vuelta entre el norte y el sur. Se trata de trasladar la idea de la existencia de vasos comunicantes entre estos dos mundos. En las clases presentamos, por ejemplo, la relación que existe entre los orígenes de la vivienda social a finales del siglo XIX en Inglaterra con las políticas de la CORVI en el Chile de principios de los años setenta; o entre las primeras experiencias cooperativistas en los países del norte de Europa en los años cincuenta y su traslado al tema de la vivienda en las Cooperativas de Construcción de Vivienda por Ayuda Mutua a finales de los años sesenta en Uruguay. Pero también, en ese camino de ida y vuelta, acercamos la influencia de estas experiencias latinoamericanas sobre algunos programas de viviendas de la Junta de Andalucía en España, como el de autoconstrucción, que se desarrolló entre los años 1988 y 2007.

En HyD nos interesa tender puentes. Tal y como propone Boaventura de Sousa Santos, es importante superar el pensamiento abismal ${ }^{9}$ por medio de la traducción intercultural y una ecología de saberes. En el caso de la arquitectura, se trata de puentes que afrontan el acercamiento entre el sur y el norte. De igual manera, siguiendo las propuestas de Edgar Morin (2004) acerca del pensamiento complejo, en la asignatura tratamos de preparar al alumno para lo inesperado: "todo lo importante que sucede en la historia mundial o en nuestra vida es totalmente inesperado, por qué continuamos actuando como si nada inesperado debiera suceder nunca [...]. El pensamiento complejo no rechaza [...] la claridad, el orden [...], sabe que no podemos programar el descubrimiento, el conocimiento, ni la acción" (Morin, 2004, p. 117). En los mismos términos se expresa Nicholas Taleb cuando afirma: "El género humano padece de una infravaloración crónica de la posibilidad de que el futuro se salga del camino inicialmente previsto" (Taleb, 2011, p. 215). Este planteamiento quedó plenamente confirmado con el estallido de la crisis de 2008. Concretamente en España el sector de la vivienda y de la construcción experimentaron un descenso del que todavía no nos hemos recuperado, casi 10 años después.

Es importante destacar que durante al menos 10 años España fue uno de los países promotores de la denominada arquitectura milagrosa (Moix, 2010). Si establecemos como puntos de partida, por un lado, la inauguración en 1997 del Museo Guggenheim de Bilbao y, por otro, la aprobación de la Ley 6/1998, de 13 de abril, sobre régimen del suelo y valoraciones, en la que se propiciaba la liberalización del suelo en torno a las ciudades, las Comunidades Autónomas y los Ayuntamientos dispusieron del ejemplo y la herramienta perfectos para la puesta en
9 “[...] líneas radicales que dividen las experiencias, los actores y los saberes sociales entre los que son visibles, inteligibles o útiles (los que quedan de este lado de la línea) y los que son invisibles, ininteligibles, olvidados o peligrosos (los que quedan del otro lado de la línea)" (Santos, 2010, p. 10). 
marcha de ese milagro que vinculaba la construcción de viviendas con el ingreso de importantes sumas de dinero en las arcas municipales. El resultado de esa mezcla se puede ver en la Ciudad de las Artes y las Ciencias de Valencia, en la Ciudad de la Cultura de Santiago o el Centro de las Artes y la Cultura de Alcorcón, en el área metropolitana de Madrid, la Ciudad de la Luz de Alicante y otros muchos edificios emblemáticos que aspiraban a repetir el éxito del Guggenheim. Paralelamente a esta acción institucional también se llevaron adelante grandes desarrollos residenciales y sobre todo grandes operaciones especulativas, así como sonados casos de corrupción, que formaron parte de la denominada burbuja inmobiliaria.

En el acercamiento al tema de la vivienda le damos mucha relevancia al papel jugado por los viviendistas, que han trazado desde hace años caminos por los que actualmente transitamos. Arquitectos como el activista Miloon Kothari, la catedrática Raquel Rolnik o el profesor Víctor Pelli, ${ }^{10}$ son algunos de estos viviendistas que desde sus respectivos países están aportando reflexiones muy interesantes para los debates en el aula. Carlos González Lobo con "Espacio máximo, costo mínimo", desde México, Edin Martínez desde Fundasal en El Salvador, ${ }^{11}$ o Joan Mac-Donald desde Chile, quien presidió durante más de diez años el Servicio Latinoamericano, Africano y Asiático de Vivienda Popular (SELAVIP).${ }^{12}$ Por señalar un ejemplo muy cercano, en diciembre de 2006 Miloon Kothari fue invitado por España como relator especial de Naciones Unidas encargado de redactar un informe "sobre una vivienda adecuada como elemento integrante del derecho a un nivel de vida adecuado". En febrero de 2008, entre sus recomendaciones, publicó lo siguiente:

94. El Estado también debería castigar con dureza prácticas tales como el acoso inmobiliario, la corrupción y la discriminación en el sector inmobiliario [...]. 95. Las autoridades a todos los niveles deberían estudiar la aplicación de los Principios básicos y directrices sobre los desalojos y el desplazamiento generados por el desarrollo, en particular la recomendación de llevar a cabo evaluaciones de los efectos de los desalojos (Kothari, 2008, p. 28).

Ese mismo año 2008 estalló en todo el mundo la crisis económica y en España, además, una grave crisis inmobiliaria. En febrero de 2009 se creó en Barcelona la Plataforma de Afectados por la Hipoteca (PAH), movimiento que reclamaba, tal y como ya sugería Kothari, una mayor protección para las personas que no podían afrontar los pagos de su hipoteca. Una de sus socias fundadoras, Ada Colau, pasó de encabezar las protestas pacíficas para evitar los desahucios a ser, tras las elecciones de mayo de 2015, alcaldesa de la propia ciudad de Barcelona. El mismo fenómeno se ha producido, en un vuelco político sin precedentes, en la mayoría de las ciudades españolas, como Madrid, Valencia o Zaragoza. Precisamente el caso de España es paradigmático en lo que se refiere a la transformación y deriva de movimientos sociales pacíficos surgidos en las plazas públicas (como el movimiento de los indignados que salieron a la calle el 15 de mayo de 2011 y quedaron acampados en las plazas de muchas ciudades) a convertirse en actores políticos que en 2015, bajo el paraguas de un partido recién creado llamado Podemos, alcanzaron el poder en muchos municipios, colocando entre otras cosas el problema de la vivienda en el primer lugar de la agenda de las instituciones locales. 


\section{Sobre el desarrollo}

A partir de las actitudes y comportamientos que podemos desplegar en nuestro encuentro con el otro (el conflicto, el aislamiento o el diálogo, a decir de Kapuscinski, 2007), desde HyD tratamos de formar a los estudiantes en la tercera de las actitudes, esto es, el diálogo y la cooperación. Se trata de mostrarles diferentes mecanismos para cooperar, ya sea a nivel profesional con la transdisciplinariedad, ya sea con las personas usuarias por medio de la participación o la mediación. Ese desarrollo tiene precisamente un componente social que intentamos transmitir a los estudiantes, por un lado, desde el enfoque más antropológico vinculado con ese encuentro con el otro, con las diferencias culturales, hasta el enfoque más social que tiene que ver con las desigualdades económicas, de raza, género o religión.

También se intenta transmitir la idea del desarrollo desde el punto de vista de las herramientas de la cooperación. Aunque se introduce la historia de la cooperación al desarrollo en materia de habitabilidad básica, cuyo origen hay que situarlo en las últimas tres décadas del siglo $\mathrm{xx}$, nos interesa especialmente trasladar una idea más general de la cooperación que propone Richard Sennett (2012) cuando diferencia una izquierda política que entiende la cooperación como un instrumento, poniendo el énfasis en los objetivos dialécticos; frente a una izquierda social, que habla de la cooperación como un fin en sí mismo, poniendo el énfasis en los procesos dialógicos (Sennett, 2012, p. 71). Por otro lado, desde la misma asignatura, discutimos la conveniencia de empezar a hablar ya de posdesarrollo, que consiste en desplazar el paradigma tecnocientífico desarrollista — que equipara crecimiento con bienestar, pero olvidando a la gente real y sus necesidades-, para insistir "en los aspectos culturales y en los valores de las sociedades sometidas a la expansión forzosa de la modernización" (Unceta, 2011, p. 47). Se busca así resolver las "anomalías" (según el citado Koldo Unceta) del "maldesarrollo": un crecimiento económico que coincide con mayor pobreza y desigualdad, deterioro ambiental, inequidad de género u olvido de los derechos humanos.

Desde el año 2002, los profesores de la asignatura hemos colaborado con la Fundación Habitáfrica (ahora llamada "Alianza por la Solidaridad") ${ }^{13}$ en diferentes proyectos que se han desarrollado en varios países africanos. Tras una primera colaboración en Mauritania, en 2008 comenzamos un proyecto de fortalecimiento institucional en la isla de Maio en Cabo Verde. En la primera etapa participamos activamente en el proyecto de mejora de las condiciones de habitabilidad en Maio, contribuyendo a la puesta en valor y la preservación de su patrimonio cultural. En concreto, por una parte se llevó a cabo la reforma o rehabilitación de 70 viviendas del centro histórico, de la entonces denominada Vila do Maio (actualmente Porto Inglés), incidiendo en la mejora de las condiciones de vida de la población más vulnerable. En segundo lugar, se elaboró un catálogo de bienes de interés arquitectónico y cultural que tuvo su culminación en 2010 con la edición del InventarioCatálogo de Patrimonio Cultural de la Isla de Maio.

Paralelamente, entre los años 2008 y 2010, el Grupo de investigación ARQPA (Arquitectura, Patrimonio, Participación) desarrollamos el proyecto La Isleta Participa, en este singular barrio de la ciudad de Las Palmas de Gran Canaria (Figuras 1 y 2). Entre 2011 y 2013, de forma simultánea, en Canarias y en Cabo Verde, impulsamos el proyecto denominado Indicadores Urbanos Activos (IURA). Ciertamente, podría-
13 Se puede acceder a los proyectos desarrollados en http:/ / www.alianzaporlasolidaridad.org/es. 


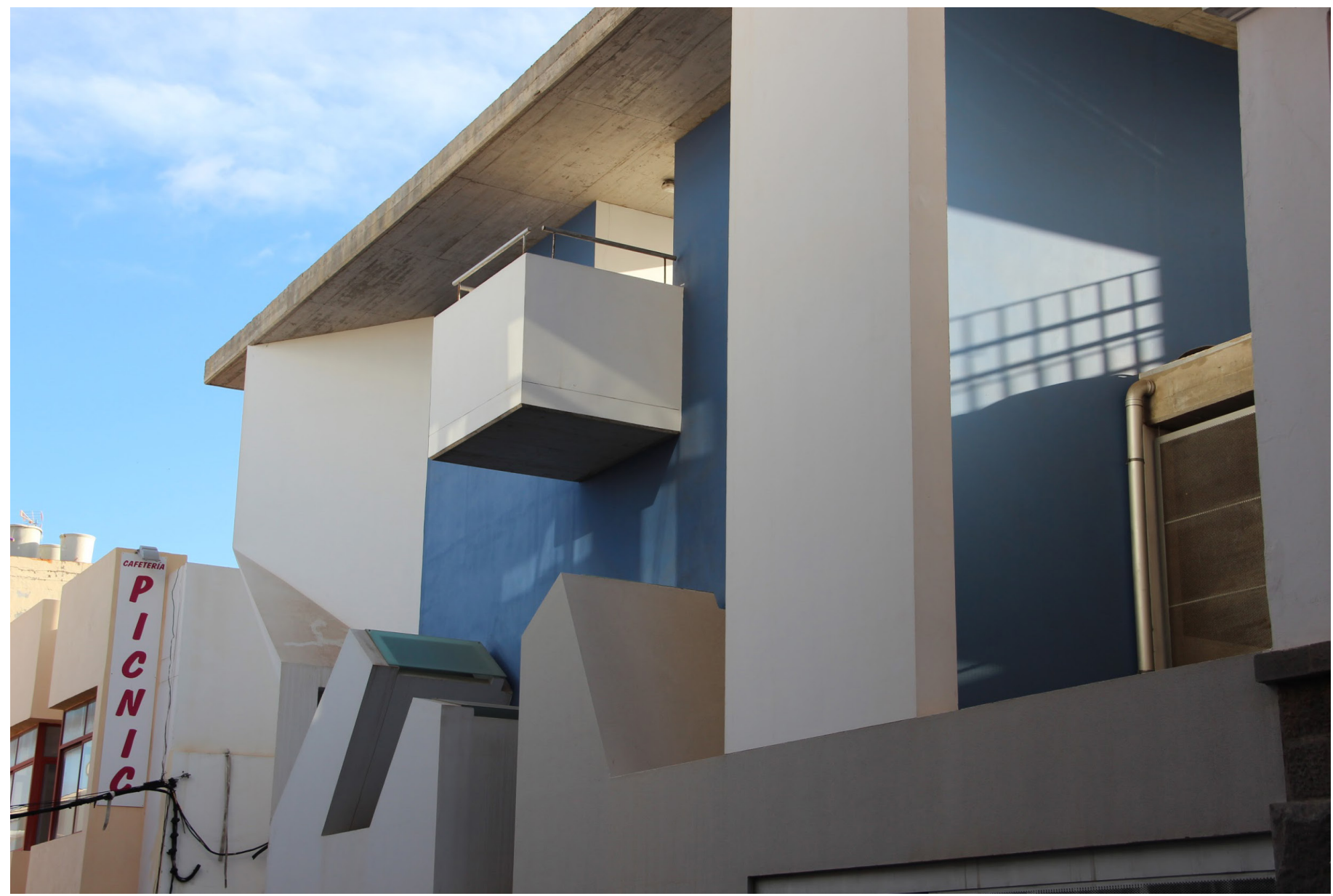

Figura 1. Barrio de La Isleta. Centro Cultural Pepe Dámaso. Centro propuesto por el proyecto de investigación La Isleta Participa. En la actualidad está destinado a fomentar la participación entre los colectivos del barrio. Fotografía de mayo de 2017. Fuente: Elaboración propia.
14 Para saber más del Centro Experimental de la Vivienda Económica de Córdoba, Argentina, se puede consultar http:/ / www.ceve.org.ar.

15 Se puede profundizar sobre

FUCVAM en http://www.fucvam.org.uy. mos trazar múltiples paralelismos entre ambas realidades, sin embargo, nuestra intención no consiste en una tarea de puesta en común o de enlace entre ambos territorios, con relaciones de hermanamiento o acuerdos entre administraciones o ciudadanías de ambos lugares. Nuestra apuesta consiste en utilizar herramientas comunes, tanto en el barrio de La Isleta como en el centro histórico de Porto Inglés. Hablamos de ciudades o barrios comunicantes, pertenecientes a una única realidad y un único mundo. Al igual que el teléfono móvil o Internet son instrumentos o herramientas que no establecen distinciones entre las partes, nuestra investigación quería avanzar en la aplicación de herramientas de análisis, urbanísticas o metodológicas comunes, que se pudieran aplicar a ambas realidades con la única adaptación propia de ser países, historias o islas diferentes.

El resultado más interesante ha sido la constatación de la existencia de un centro vivo cuya preservación pasa por la participación ciudadana en el conocimiento y la protección activa de los valores existentes, entre los que queremos destacar la que denominamos arquitectura habitual, esto es, la arquitectura relacionada con el hábitat, el habitar, los hábitos y los habitantes. Se introducen ejemplos de proyectos de cooperación al desarrollo, pero también se presentan experiencias como la del Centro Experimental de la Vivienda Económica (CEVE) de Córdoba, Argentina, ${ }^{14}$ o la de la Federación Uruguaya de Cooperativas de Vivienda (FUCVAM). ${ }^{15}$ 


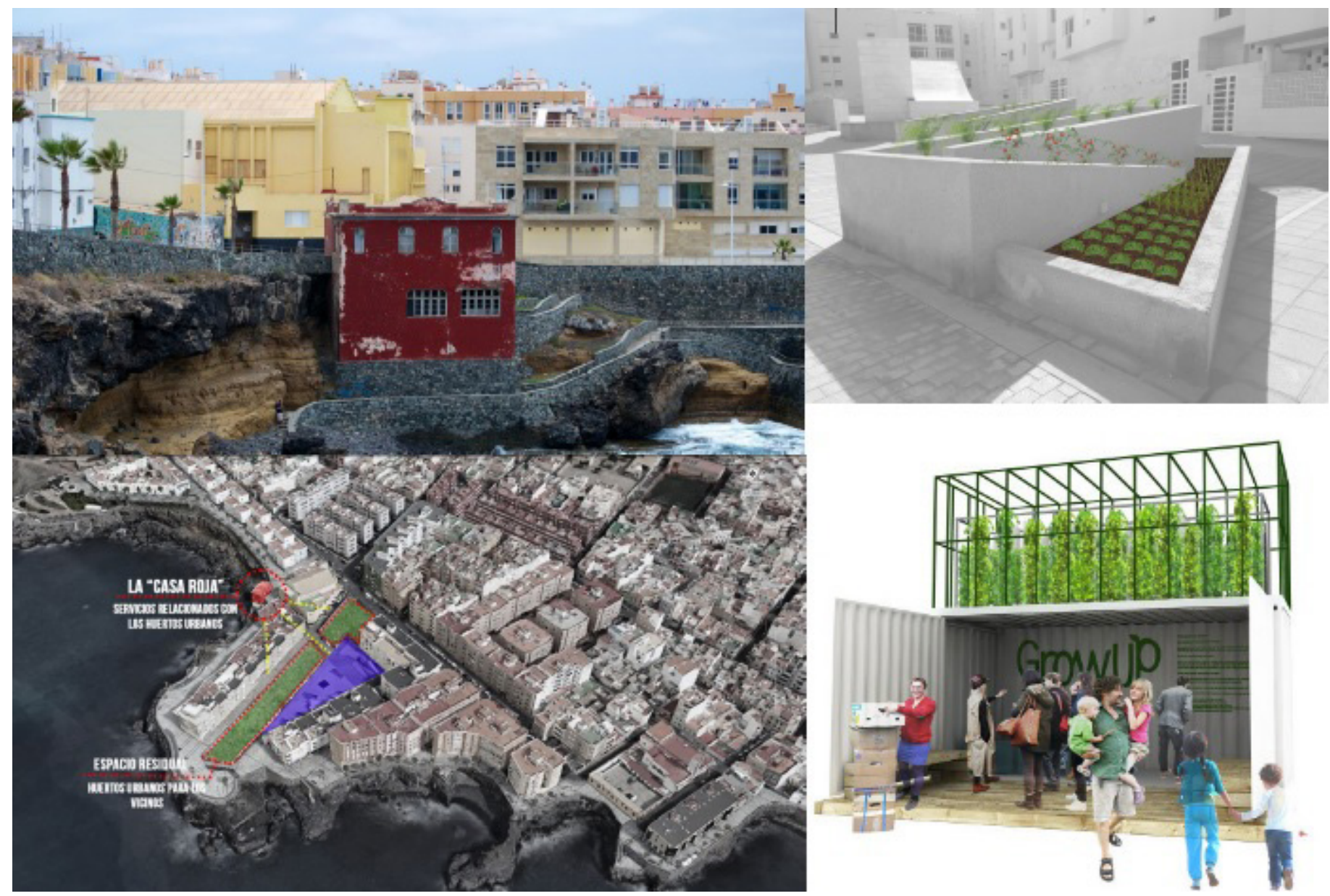

\section{Metodología}

Las clases de HyD se desarrollan durante el primer cuatrimestre en horario de tarde (tres horas y cuarenta y cinco minutos) un día por semana. Aproximadamente la mitad de las clases transcurre en el aula de la escuela de arquitectura y la otra mitad se desarrolla en diferentes espacios de la ciudad. En cuanto a las clases del aula, también se dividen en dos: una parte teórica y una parte práctica que combina la presentación de documentos audiovisuales o sonoros y el aprendizaje de las técnicas participativas.

Por ejemplo, en los cursos 2007-08 y 2008-09 propusimos como trabajo final de curso la elaboración de un documento audiovisual de un minuto de duración ${ }^{16}$ en el que los y las estudiantes debían trasladar al vecindario del barrio de La Isleta o a los usuarios del sistema de bicicleta pública algún mensaje que vinculara el lenguaje de la arquitectura que ellos estaban aprendiendo, con el lenguaje del sentido común. El resultado fue muy interesante, ya que los estudiantes pudieron poner en práctica la capacidad de síntesis y el análisis de un problema para luego plasmarlo en una composición más publicitaria que proyectual. En el curso 2014-2015 impulsamos la elaboración de una revista digital relacionada con diferentes temas (mujer, diversidad multifuncional) y diferentes ciudades del continente africano (Figura 3 ).

Tras su conversión en asignatura optativa del grado hemos adoptado definitivamente la presentación audiovisual como parte esencial del acercamiento que realizan los estudiantes a los ciudadanos. Estas "galeanas audiovisuales" nos remiten a prácticas muy conocidas a lo largo de la historia, como bien nos recuerda Richard Sennett: "Dialógica es
Figura 2. Barrio de La Isleta. Fuente: Curso 2016-2017, alumno Sergio Pérez Carballo. Intervención de recualificación en huecos urbanos.
16 Llamamos "galeana audiovisual" a una pequeña composición audiovisual de menos de 3 minutos de duración. Sigue la idea de las denominadas "galeanas", que constan de una o varias imágenes y un breve texto con el que se quiere llamar la atención sobre un aspecto muy puntual que invite a la reflexión. Recibe el nombre del escritor uruguayo Eduardo Galeano (1940-2015), por su forma de contar historias o anécdotas en obras como El libro de los abrazos o Espejos. Son muchos los escritores que utilizan esta fórmula ligera de trasladarnos una idea como Julio Cortázar, Oliverio Girondo o Augusto Monterroso. Otro autor que cultiva esta escritura imaginada es el español Juan José Millás. 


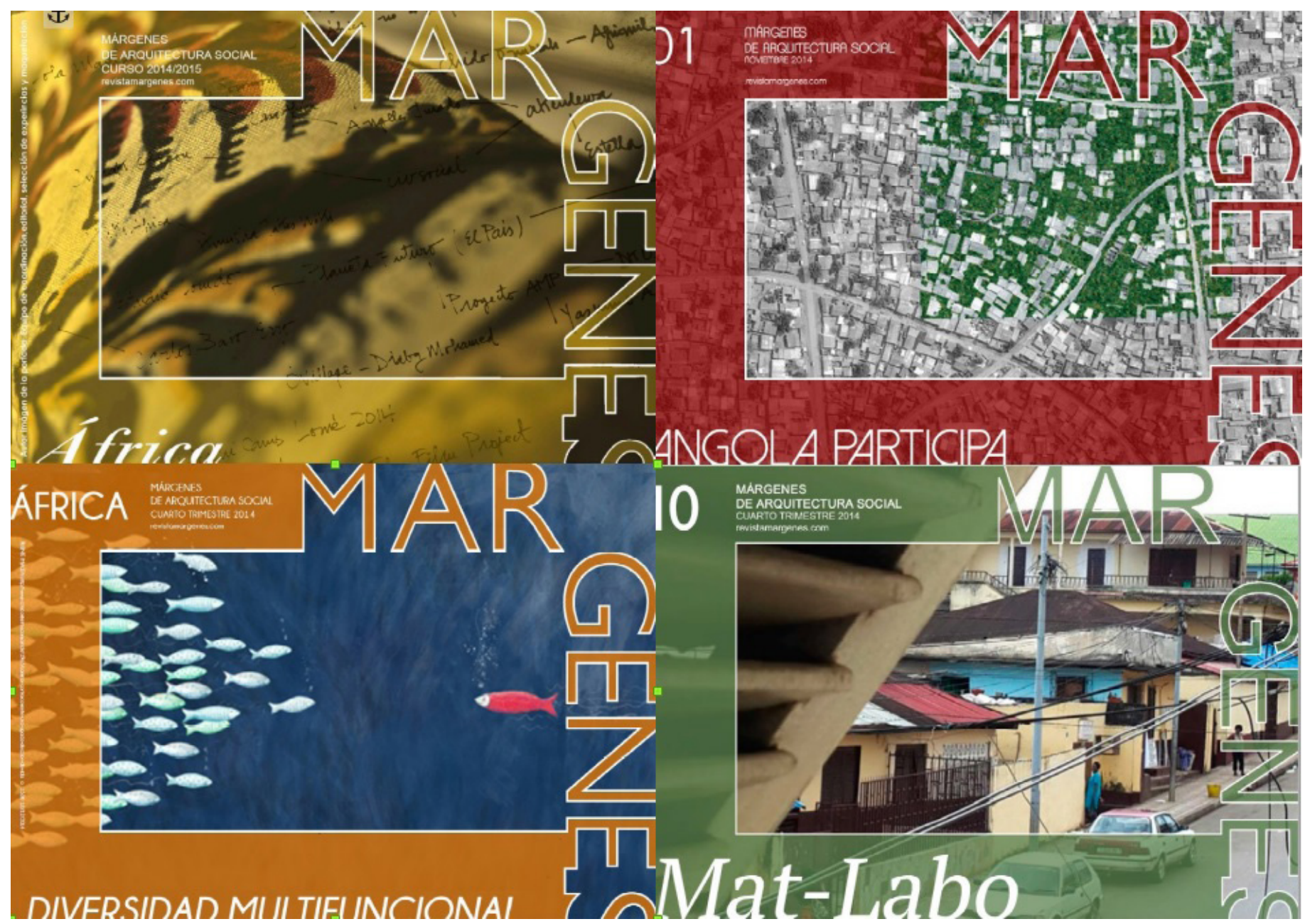

Figura 3. Portadas de algunas de las revistas de divulgación diseñadas por los alumnos en el curso 2014-2015. La revista Márgenes de Arquitectura Social tiene su origen en la Escuela de Arquitectura de Las Palmas de Gran Canaria en el año 2001 . Fuente: http://www.revistamargenes.com. en realidad la denominación moderna de una práctica narrativa muy antigua, que utilizó ya el historiador griego Heródoto al crear un mosaico de fragmentos de texto que, como los ensayos de Montaigne, producen una forma única, amplia y coherente" (Sennett, 2012, p. 388).

\section{La participación}

La participación se propone como un eje transversal en la asignatura. Primero se introduce la historia reciente de la participación en la arquitectura y su influencia en el momento presente. Se presenta la historia de la participación en la arquitectura que se desarrolló en los denominados largos años sesenta (entre los años 1957 y 1973) a través de cinco miradas (social, cultural o antropológica, intelectual, común y ecológica). Analizamos el modo cómo en esos años arquitectos como Ralph Erskine, Lucien Kroll, Christopher Alexander, Aldo Van Eyck, John Turner, Nicholas Habraken o Giancarlo de Carlo desarrollaron diferentes mecanismos de participación en la creación del hábitat.

Entre los años 2008 y 2010 desarrollamos un proyecto de investigación denominado "La Isleta Participa". Fue un proyecto silencioso, que no se planteaba en las claves de la investigación-acción-participación, sino como una investigación aplicada en el campo del urbanismo. Desde la universidad y desde el urbanismo quisimos intervenir en la realidad de uno de los barrios más singulares de la ciudad de Las Palmas de Gran Canaria. Paralelamente, en esos años, junto con las clases de $\mathrm{HyD}$, coordinábamos Proyectos Final de Carrera (PFC) que se desa- 
rrollaban en África y América latina y colaborábamos activamente en proyectos de cooperación al desarrollo. En parte fue esta mezcla de arquitectura, universidad y cooperación la que contribuyó a la obtención en el año 2010 del segundo premio Ciudad de Las Palmas de Participación Ciudadana y nos llevó en el año 2013 a obtener el premio de ensayo convocado por Casa África con el trabajo titulado Un modo de afrontar la ciudad africana (2014).

Nos gustaría creer que la participación está regresando a las escuelas de arquitectura, pues a partir del año 2008 - y coincidiendo con la crisis inmobiliaria de la que alguna culpa tendría la profesión de arquitecto- empieza a cuestionarse la existencia de un único tipo de arquitecto como "creador", aceptando otras formas de entender la disciplina con la incorporación de aspectos ambientales, sociales, transdisciplinares, etc. Sin embargo, es significativo que en la adaptación al proceso de Bolonia de los estudios de arquitectura (grado en arquitectura) se hayan reducido las materias que pueden abordar estos aspectos. A lo largo del curso intentamos formar a los estudiantes en técnicas de participación ciudadana que puedan utilizar en su quehacer profesional y que en principio no requerirían el apoyo de otros técnicos especialistas en esta materia. Se abordan nociones básicas y recursos mínimos sobre dinámicas participativas que no pretenden suplantar la labor de dichos profesionales, sino conocer la importancia de este trabajo de mediación con vecinos, usuarios o clientes, y que van desde la presentación inicial de la asignatura hasta el aprendizaje de dinámicas simples de información como la toma de datos, la lluvias de ideas o el análisis DAFO, pasando por técnicas de diagnóstico como los mapas de actores o los sociogramas, hasta técnicas más complejas derivadas de la investigación acción participativa (IAP).

Hoy es más evidente que nunca "la necesidad de que el ciudadano forme parte sustancial del sistema de toma de decisiones sobre la organización del territorio, en el que vive o en el que va a vivir" (Fariña, 2015, p. 74). Si bien no parece próximo el momento, planteado por Fariña, en el que "el propio plan como documento normativo será sustituido, por ejemplo, por una oficina de planeamiento que vaya configurando la organización territorial y urbana en tiempo real" (ob. cit., p. 77), es importante que se empiecen a dar los pasos en una descentralización administrativa que incorpore dicha participación ciudadana en todas las fases del proceso. Tras la concepción normativa del urbanismo surgida de las propuestas de los CIAM, al menos desde los años cuarenta, pasando por la concepción estratégica del planeamiento que alcanzó su punto álgido en los años noventa (Martínez, 2005), podemos estar entrando en esta segunda década del siglo XXI en una concepción participativa del urbanismo.

\section{La producción social del hábitat}

Uno de los planteamientos básicos que utilizamos en la asignatura es la idea del arquitecto como traductor del lenguaje de la arquitectura al resto de la ciudadanía y viceversa. Si reconocemos que existe un alejamiento entre la arquitectura y la sociedad, es también comprensible que exista un problema de comunicación entre los profesionales de la arquitectura y esa ciudadanía. Este alejamiento llegó a su punto álgido también en el año 2008, en el que la maquinaria de la construcción en España impedía cualquier posibilidad de diálogo entre los arquitectos 


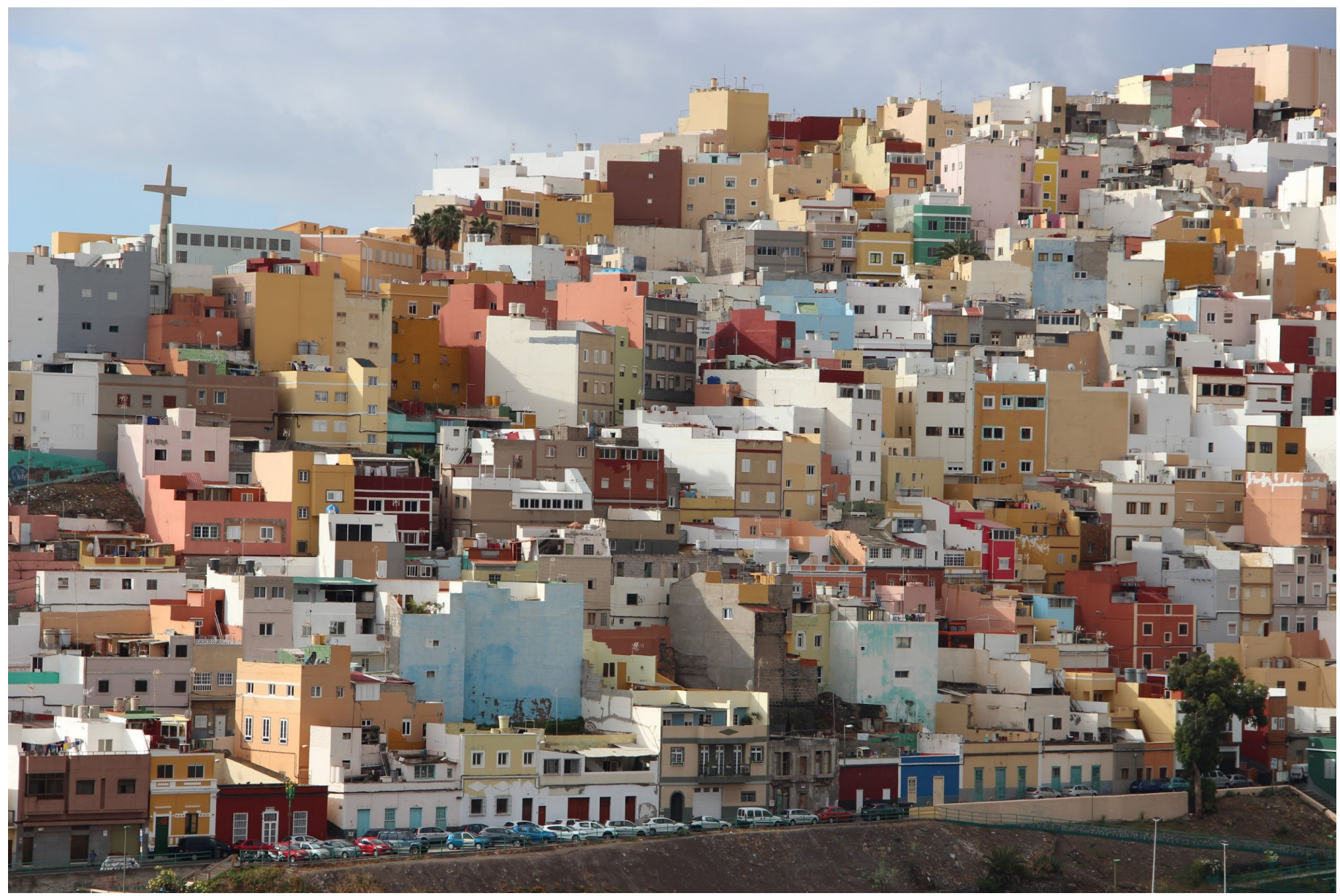

Figura 4. Risco de San Juan. Alrededores del barrio de Vegueta en Las Palmas de Gran Canaria, mayo de 2017. Fuente: Elaboración propia
17 "Lo que ocurre en el que mira, recorre y vive una arquitectura: eso es arquitectura; de esta manera - mirando, recorriendo "el mundo", viviendo, en suma- es como se aprende arquitectura. Lo demás será oficio, dibujo, construcción, urbanística..." (Martín, 1997, p. 28). y sus clientes. Paradójicamente, es la propia administración la que no contempla entre sus protocolos de intervención un lugar para la mediación o la participación en los procesos de diseño, construcción y entrega de promociones de viviendas. Todo el discurso acerca de la Producción Social del Hábitat (PSH), como corolario del propio curso, se alimenta de las tesis sobre "el derecho a la ciudad" y continúa con la larga serie de publicaciones y prácticas recogidas por Habitat Internacional Coalition (HIC) y algunos autores importantes como Gustavo Romero y Rosendo Mesías, para quienes la PSH "integra diversos factores: acceso al suelo, dotación de servicios y equipamiento suficiente, acceso a materiales y componentes, asistencia técnica, financiación y acceso a recursos, así como — de manera fundamental- la participación de los usuarios en las distintas etapas de producción" (Romero y Mesías, 2004, p. 31)

El carácter optativo de la asignatura Hábitat y desarrollo permite introducir de manera muy visible aspectos que no han sido considerados a lo largo de los cursos precedentes. Una de esas propuestas diferenciadoras consiste en realizar recorridos temáticos o itinerarios por la ciudad. ${ }^{17}$ Se trata de avanzar en la educación de la mirada de los estudiantes por medio de paseos por diferentes barrios de la ciudad de Las Palmas de Gran Canaria (Figura 4). En muchos casos estos paseos son, especialmente para los estudiantes extranjeros, una oportunidad para conocer la ciudad y sus espacios más representativos desde diferentes puntos de vista. A medida que han pasado los años, algunos recorridos se han ido consolidando - como los que realizamos anualmente a los Riscos de San Nicolás y San Antonio o por el barrio de La Isleta-, mientras que otros han ido variando o se han ido relacionando o vincu- 
lando con otras actividades en la ciudad. También es obligada la visita a Casa África, institución de ámbito nacional que se encuentra ubicada en Las Palmas de Gran Canaria.

\section{Resultados y conclusiones}

En los últimos 16 años, la asignatura optativa Hábitat y desarrollo, a tenor de lo expresado por el propio alumnado, ha sido una propuesta innovadora y diferenciada con respecto al resto de la formación que han recibido en sus años de carrera. Esta diferencia, en primer lugar, tiene que ver con el acercamiento que se realiza a otras disciplinas, como la antropología, la sociología o la geografía, que, progresivamente, han visto reducida su presencia - e incluso han sufrido un claro desprecio- en la carrera; en segundo lugar, a la novedad de las clases fuera del aula que permiten conocer la ciudad — a veces la menos transitada y marginada - y nos permiten interactuar de otra forma entre profesores y estudiantes; y por último, en tercer lugar, al acercamiento, al menos teórico, al usuario, vecino o cliente a través del aprendizaje de técnicas participativas en una concepción del arquitecto más como traductor, mediador o facilitador que como creador.

Nuestra experiencia en el desarrollo de esta asignatura nos ha llevado a entender que existe un campo muy amplio (la arquitectura para el otro 90\%) en el que la formación del arquitecto tiene mucho que aportar. Asistimos a un lento y progresivo retroceso de las pocas sociedades del bienestar que han sido (principalmente en Europa); a la sectorización, e incluso fortificación, de amplios sectores de las grandes ciudades en respuesta a la inseguridad; la masificación de las grandes ciudades del mundo, superando con creces todas aquellas previsiones catastrofistas de los años setenta (Metro Manila, Saigón, Ciudad de México, etc.); al desarrollo de modelos insostenibles de ciudad dispersa absolutamente dependientes de los combustibles fósiles, que además encarecen y en muchos caso imposibilitan el acceso a los servicios de agua o saneamiento. Cuando ya conocemos los efectos nocivos de estos problemas, ahora más que nunca nos corresponde aportar nuevas respuestas y, ojalá, verdaderas soluciones.

En HyD proponemos a los estudiantes dejar aparcada por un momento la idea del arquitecto como creador o como artista para adoptar por un instante la idea del arquitecto como artesano, "como traductor de deseos y necesidades; mediador y facilitador de procesos; catalizador de situaciones o conector entre sujetos e intereses" (Verdaguer, 2011). Esto nos permite integrarnos, por ejemplo, en procesos de construcción o de reposición de viviendas como interlocutores entre los diferentes actores: beneficiarios, técnicos, políticos, etc. Proponemos también poner en práctica la capacidad para gestionar la complejidad. Esa capacidad adquirida a lo largo de los cursos de proyectos, urbanismo, construcción o instalaciones, y que nos permite integrarnos en equipos transdisciplinares con herramientas muy válidas para afrontar la toma conjunta de decisiones.

Por último, queremos referirnos a la arquitectura social como aquella arquitectura que pone a las personas, a las comunidades y a la sociedad por delante del objeto arquitectónico. Durante los años previos a la crisis se puso claramente en evidencia que existía una arquitectura de la imagen y del espectáculo, relacionada con arquitectos estrella (Sudjic, 2007), que ignoraba importantes variables como la economía, 
la sostenibilidad, la participación ciudadana o la ecología. Sin embargo, en el otro extremo, por ejemplo, en el sur o en la cooperación al desarrollo, las prácticas arquitectónicas incorporaron una serie de variables relacionadas con la sociedad a la que servían. Los materiales, las tecnologías, los procesos o los instrumentos respondían a otras lógicas locales, integradoras y vinculadas a las necesidades básicas y sus satisfactores. Esa arquitectura social propone por un lado un acercamiento de nuestra disciplina al resto de disciplinas técnicas y sociales, pero también propicia una apuesta decidida por el diálogo con la sociedad, con los habitantes de las ciudades. Ya no se trata de un problema que afecta a las capas más pobres o marginadas. Todos y todas estamos implicados en el problema planteado en este nuevo milenio por esa ciudad considerada ya como indiscutible hábitat del ser humano.

Si bien el origen de la formación en cooperación al desarrollo en las escuelas de arquitectura de España tuvo lugar simultáneamente a la celebración, en 1996, de la Segunda Conferencia de las Naciones Unidas sobre los Asentamientos Humanos (Hábitat II) en Estambul, Turquía, tenemos que decir que, más de dos décadas después, ni la profunda crisis económica del año 2008, ni la aprobación en el año 2000 de los Objetivos del Milenio y en el 2015 de los Objetivos de Desarrollo Sostenible, ni siquiera la celebración en 2016 en Quito, Ecuador, de la Tercera Conferencia de las Naciones Unidas sobre los Asentamientos $\mathrm{Hu}$ manos (Hábitat III), han tenido suficiente influencia para incorporar estos contenidos en el corpus central de la disciplina. Sin duda, a los que estamos convencidos de su importancia, nos corresponde redoblar esfuerzos y argumentos para que dicho cambio se produzca cuanto antes, por el bien del alumnado y por el bien de la arquitectura. 


\section{Referencias}

Alexander, C. et al. (1980). Un lenguaje de patrones: ciudades. Edificios. Construcciones. Barcelona: Gustavo Gili.

Ascher, F. (2010). Los nuevos principios del urbanismo. Madrid: Alianza.

Baricco, A. (2012). Mr. Gwyn. Barcelona: Anagrama.

Blundell, P., Petrescu, D. y Till, J. (2005). Architecture and participation. Londres: Taylor y Francis.

De Manuel, E. (2010). Construyendo triángulos para la gestión social del hábitat. Hábitat y sociedad, 1, 13-37.

Ettlinger, L. (1984). La aparición del arquitecto italiano durante el siglo Xv. En Kostof, S. (coord.), El arquitecto: historia de una profesión. Madrid: Cátedra.

Fariña Tojo, J. (2015). Cambiar el modelo urbano. Ciudades, 18 (1), 69-79.

Florida, R. (2009). Las ciudades creativas. Barcelona: Paidós.

Franco, J.T. (2016). ¿Cómo los arquitectos daneses "conectan" con los usuarios para entregarles la mejor arquitectura posible? Recuperado el 8 de julio de 2017 de: http:/ /www.archdaily.mx/mx/773423/ como-los-arquitectos-daneses-conectan-con-losusuarios-para-entregarles-la-mejor-arquitectura-posible.

Giddens, A. (1999). Consecuencias de la modernidad. Madrid: Alianza editorial.

Holston, J. (1995). Spaces of Insurgent Citizenship. Planning Theory, 13, 37-56.

Jacobs, J. (1961). Vida y muerte de las grandes ciudades americanas. Madrid: Capitán Swing.

Kapuscinski, R. (2007). Encuentro con el otro. Barcelona: Anagrama.

Kothari, M. (2008), Informe del Relator Especial sobre una vivienda adecuada como elemento integrante del derecho a un nivel de vida adecuado. Misión a España. Consejo de Derechos Humanos, Séptimo período de sesiones, tema 3 del programa. Recuperado el 8 de julio de 2017 de: http://www2.ohchr.org/english/bodies/hrcouncil/docs/7session/A-HRC-7-16-Add2_ sp.doc.

Latouche, S (2011). La hora del decrecimiento. Barcelona: Octaedro.

Lorenzo, P. (coord.) (2005). Un techo para vivir: tecnologías para viviendas de producción social en América latina. Barcelona: UPC.

Martín Hernández, M. (1997). La invención de la arquitectura. Madrid: Celeste.

Martín, M., Díaz, V. y Rodríguez, E. (2014). Un modo de afrontar la ciudad africana. Madrid: Los libros de la Catarata/Casa África.

Martínez López, M. (2005). Urbanismo, participación ciudadana y planificación estratégica de ciudades. Recuperado el 8 de julio de 2017 de: http:/ / www.
miguelangelmartinez.net/IMG/pdf/2005_Plan_estrategica_ciudades.pdf.

Max-Neef, M. (1994). Desarrollo a escala humana. Conceptos, aplicaciones y algunas reflexiones. Barcelona: Icaria.

Moix, L. (2010). Arquitectura milagrosa. Hazañas de los arquitectos estrella en la España del Guggenheim. Barcelona: Anagrama.

Montaner, J.M. y Muxí, Z. (2011). Arquitectura y política. Ensayos para mundos alternativos. Barcelona: Gustavo Gili.

Montes Serrano, C. (2000). El CIAM IV y la Carta de Atenas. La contribución inglesa y los inicios del grupo MARS. En AA.VV, Forma Urbis. Pamplona: Universidad de Navarra, pp. 185-195.

Morin, E. (2004). Introducción al pensamiento complejo. Barcelona: Gedisa.

ONU (2015). Objetivos de desarrollo sostenible. Recuperado el 8 de julio de 2017 de: http:/ / www.un.org/sustainabledevelopment/es/objetivos-de-desarrollosostenible.

Pallasmaa, J. (2015). Habitar en el tiempo. Habitar. Barcelona: Gustavo Gili.

Pelli, V. (2006). Habitar, participar, pertenecer. Acceder a la vivienda-incluirse en la sociedad. Buenos Aires: Nobuko.

Romero, G. y Mesías, R. (coord.) (2004). La participación en el diseño urbano y arquitectónico en la producción social del hábitat, México D.F.: CYTED-HABYTED.

Rudofsky, B. (1973). Arquitectura sin arquitectos: breve introducción a la arquitectura sin genealogía. Buenos Aires: Editorial Universitaria.

Salas, J. (1998). Contra el hambre de vivienda: soluciones tecnológicas latinoamericanas. Bogotá: Escala.

Santos, B. de S. (2010). Para descolonizar Occidente. Más allá del pensamiento abismal. Buenos Aires: CLACSO.

Sassen, S. (2010). Una sociología de la globalización. Madrid: Katz editores.

Secchi, B. (2015). La ciudad de los ricos y la ciudad de los pobres. Madrid: Los libros de la Catarata.

Sennett, R. (2012). Juntos. Rituales, placeres y política de cooperación. Barcelona: Anagrama.

Sennett, R. (2009). El artesano. Barcelona: Anagrama.

Steffen, W. et al. (2015). The trajectory of the Anthropocene: The Great Acceleration. Anthropocene Review, 2 (1), 81-98.

Sudjic, D. (2007). La arquitectura del poder. Barcelona: Arial.

Taibo, C. (2017). En defensa del decrecimiento. Sobre capitalismo, crisis y barbarie. Madrid: Los libros de la Catarata.

Taleb, N. (2011). El cisne negro. El impacto de lo altamente improbable. Barcelona: Paidós. 
Turner, J. (1977). Vivienda, todo el poder para los usuarios: hacia la economía en la construcción del entorno. Madrid: Blume.

Unceta Satrústegui, K. (2011). ¿Del desarrollo al postdesarrollo?: Propuestas para un debate necesariamente transdisciplinar. En Matarán, A. y López, F., La Tierra no es muda: Diálogos entre el desarrollo sostenible y el postdesarrollo. Granada: Universidad de Granada, pp. 25-68.
Verdaguer, C. (2011). Marco conceptual: La participación ciudadana como instrumento de sostenibilidad urbana. Recuperado el 8 de julio de 2017 de: http://www.diba.cat/c/document_library/get_file?uuid=22bff79a-64a2-4b22-9f11$05 d 1435 d 547$ a\&groupId=527890.

Zweig, S. (2002). Momentos estelares de la humanidad. Barcelona: El Acantilado.

Martín Hernández, M.J. y Díaz garcía, V.J. (2017). Hábitat y desarrollo. Explorando caminos de innovación educativa en arquitectura y urbanismo. Hábitat y Sociedad, 10, 113-128.

<http://dx.doi.org/10.12795/HabitatySociedad.2017.i10.07> 